\section{RSP}

http://www.rsp.fsp.usp.br/
Revista de Saúde Pública

\title{
O papel da literacia em saúde como fator associado às perdas dentárias
}

\author{
Carla Fabiana Tenani' (iD, Manoelito Ferreira Silva Junior" (iD), Carolina Matteussi Lino' (iD, Maria \\ da Luz Rosário de Sousa ${ }^{\text {III }}$ iD, Marília Jesus Batista ${ }^{\text {III,IV iD }}$ \\ I Universidade Estadual de Campinas. Faculdade de Odontologia de Piracicaba. Programa de Pós-Graduação \\ em Odontologia. Piracicaba, São Paulo, Brasil \\ " Universidade Estadual de Ponta Grossa. Faculdade de Odontologia. Departamento de Odontologia. Ponta \\ Grossa, Paraná, Brasil \\ III Universidade Estadual de Campinas. Faculdade de Odontologia de Piracicaba. Departamento de Ciências da \\ Saúde e Odontologia Infantil. Piracicaba, São Paulo, Brasil \\ Iv Faculdade de Medicina de Jundiaí. Departamento de Saúde Coletiva. Jundiaí, São Paulo, Brasil
}

Correspondência:

Marília Jesus Batista Avenida Limeira, 901 13414-018 Piracicaba, SP, Brasil E-mail: mariliamota@g.fmj.br

Recebido: 18 jan 2021

Aprovado: 6 abr 2021

Como citar: Tenani CF, Silva-Junior MF, Lino CM, Sousa MLR, Batista MJ. O papel da literacia em saúde como fator associado às perdas dentárias. Rev Saude Publica. 2021;55:116.

https://doi.org/10.11606/

s1518-8787.2021055003506

Copyright: Este é um artigo de acesso aberto distribuído sob os termos da Licença de Atribuição Creative Commons, que permite uso irrestrito, distribuição e reprodução em qualquer meio desde que o autor e a fonte originais sejam creditados.

\section{RESUMO}

OBJETIVO: O objetivo foi analisar o papel da Literacia em Saúde (LS) como fator associado às perdas dentárias entre usuários do Sistema Único de Saúde com doenças crônicas não transmissíveis.

MÉTODOS: O estudo transversal e analítico foi conduzido com usuários adultos e idosos selecionados em dez Unidades de Saúde da Família sorteadas, em Piracicaba - SP, Brasil. Foi aplicado um questionário com dados sociodemográficos (sexo, idade, cor da pele e escolaridade), comportamentais (escovação e uso de fio dental), determinantes em saúde (tipo e frequência de uso de serviço de saúde médico e odontológico) e clínica (dor). As condições bucais foram coletadas por exame intrabucal do biofilme dental visível e Índice Pediodontal Comunitário. As condições clínicas sistêmicas (glicemia, hemoglobina glicada e pressóricas) foram extraídas dos prontuários. A variável explanatória foi a LS (baixa, média e alta), medida pelo Health Literacy Scale (HLS-14).

RESULTADOS: O desfecho foi à perda dentária medida pelo Índice de dentes permanentes cariados, perdidos e obturados. Foi realizada regressão logística com uso de um modelo conceitual para a LS ( $<<0,05)$. Para os 238 indivíduos, a média de idade foi 62,7 anos $( \pm 10,55)$. A perda dentária esteve associada à LS nos modelos de regressão ajustados por tipo de serviço odontológico, frequência odontológica e uso de fio dental. No modelo final, a perda dentária teve como fatores associados a maior idade (OR $=1,12$; IC95\% 1,07-1,17), menor escolaridade $(\mathrm{OR}=3,43$; IC95\% 1,17-10,10), ao uso irregular de fio dental (OR = 4,58; IC95\% 1,75-7,31), uso irregular do serviço odontológico (OR = 2,60; IC95\% 1,32-5,12), bolsa periodontal (> 4mm) (OR = 0,31; IC95\% 0,01-0,08), ter biofilme dental visível (OR = 7,23; IC95\% 3,19-16,41) e maior índice de glicemia (OR = 1,98; IC95\% 1,00-3,92).

CONCLUSÕES: A perda dentária esteve associada à LS quando ajustada por comportamentos em saúde, a partir da inclusão das variáveis sociodemográficas e condições clínicas ela perdeu a significância. No modelo final, comportamentos, determinantes em saúde e condições clínicas foram indicadores de risco da perda dentária, demonstrando a multifatorialidade envolvida neste fenômeno.

DESCRITORES: Saúde Bucal. Índice CPO. Conhecimentos, Atitudes e Prática em Saúde. Educação em Saúde Bucal. 


\section{INTRODUÇÃO}

As Doenças Crônicas Não Transmissíveis (DCNT) caracterizam-se por sua etiologia multifatorial e estão associadas a diversos fatores de risco ${ }^{1}$. Estima-se que, em 2020, só nas Américas, 61 milhões de pessoas tinham diabetes e, em todo o mundo, 70,9 milhões de pessoas morreram no ano de 2019, em decorrência da diabetes mellitus ${ }^{3}$. Outro fator de risco importante é a hipertensão, que afetou mais de um bilhão de pessoas só no continente americano ${ }^{4}$, em 2019, a pressão arterial sistólica foi o principal fator global de óbitos entre indivíduos acima de 50 anos $^{2}$. Esses números levam à constatação da urgente necessidade de estratégias para o enfrentamento e prevenção dessas condições.

Atualmente, nas Américas, 81\% das mortes ocorrem por DCNT que 74\% das mortes foram causadas pelo mesmo motivo 5 . Só em São Paulo, em 2017, 65\% das mortes em todo o estado foram em decorrência de $\mathrm{DCNT}^{6}$, e diversos outros estudos demonstram a procura de pacientes com doenças crônicas por serviços emergenciais e hospitalizações ${ }^{7,8}$.

Outro grande desafio à saúde da população são as condições bucais ${ }^{9}$, principalmente a perda dentária, classificada em 2019 como a 22a maior causa de deficiência em saúde, $31^{\mathrm{a}}$ em prevalência e $56^{\mathrm{a}}$ em incidência ${ }^{10}$. A cárie e a doença periodontal, além de outros comportamentos em saúde, são os principais fatores de risco conhecidos para a perda dentária ${ }^{11}$, no entanto, estudos demonstram que perdas dentárias têm sido associadas a alterações sistêmicas, como risco cardíaco, por exemplo, evidenciando a necessidade de estratégias com abordagens integrais do cuidado $^{12}$, nesse sentido, a Organização Mundial da Saúde (OMS) tem destacado a Literacia em Saúde (LS) como uma chave importante para promoção da saúde, considerada um fator mensurável e modificável.

Ao contrário da LS, os determinantes estruturais de saúde são mais difíceis de serem modificados, a literacia pode ser alterada por meio de intervenções de promoção de saúde, educação em grupo, entrevistas motivacionais e aconselhamentos, aumentando a autonomia nas tomadas de decisão. A modificação dos níveis de literacia pode ser mensurada por meio de instrumentos validados de fácil aplicação para abordar o paciente, individual ou coletivamente $e^{13,14}$.

A LS é a capacidade de obter e compreender informações básicas necessárias para tomar decisões em saúde, abrangendo componentes fundamentais na busca por bem-estar e na promoção da saúde, mostrando-se um importante marcador de desigualdade ${ }^{15,16}$. Como campo de pesquisa a LS vem ganhando destaque, seja como fator de interferência em comportamentos e condições de saúde ${ }^{17,18,19}$, seja na transição epidemiológica com aumento das DCNT. Há, atualmente, muita facilidade em se obter informações de saúde, principalmente por meio da internet, porém, muitas dessas informações são errôneas, principalmente graças à disseminação indiscriminada de notícias falsas, que promovem desinformação e afetam a saúde da população, inclusive ameaçando vidas ${ }^{20}$. Nesse sentido, a LS pode ser de grande relevância para determinar a tomada de decisões em saúde ${ }^{15}$, ainda mais quando controlada e direcionada por fatores como idade, renda, emprego, nível educacional e cor da pele.. A literatura recente revela que indivíduos com níveis baixos de LS têm menos tempo de vida, mais carga de doenças, incapacidade de uso de serviço de saúde e geram mais custos aos serviços ${ }^{21}$.

Diante do exposto, é relevante verificar o papel da LS nas perdas dentárias, a partir de um modelo conceitual teórico, no contexto das DCNT. Assim, o objetivo deste estudo foi analisar o papel da LS como fator associado às perdas dentárias entre usuários do Sistema Único de Saúde (SUS) portadores de DCNT.

\section{MÉTODOS}

\section{Desenho e Local do Estudo}

Estudo transversal e analítico, realizado sob o Strengthening the Reporting of Observational Studies in Epidemiology (STROBE) para estudos transversais ${ }^{22}$, na cidade de Piracicaba, São 
Paulo, Brasil, com amostra aleatória entre usuários de Unidades de Saúde da Família (USF) na Atenção Primária em Saúde (APS) do Sistema Único de Saúde (SUS).

\section{Universo e Amostra}

Segundo o Censo em 2010, a população estimada de Piracicaba era de 364.571 residentes na área urbana e a população adulta e idosa correspondia a $261.567^{23}$. A rede municipal de saúde contava com 71 Unidades Básicas de Saúde, sendo 51 USF $^{24}$.

Para as Unidades de Saúde, considerou-se o estudo de Morgan $(2013)^{26}$ e foi determinado o número de 8 USF e quatro suplentes, dentre as 51 USF de Piracicaba.

Para os indivíduos, o tamanho da amostra foi calculado considerando a prevalência da alta LS como $50 \%^{25}$, erro de $10 \%$ e efeito de delineamento dois. A amostra final estimada para o estudo foi de 238 indivíduos. Estimando uma provável perda, foi acrescentado $20 \%$ chegando ao total de 298 indivíduos.

\section{Seleção da amostra}

Para a seleção das Unidades de Saúde, realizou-se um sorteio de 8 Unidades e mais 4 suplentes ${ }^{26}$, de forma probabilística, considerando o número de hipertensos e diabéticos cadastrados por Unidade no sistema informatizado do município. Após duas recusas, incluíram-se duas USF suplentes sorteadas, porém, devido à dificuldade em atingir o número de usuários em algumas USF, foram incluídas outras 2 suplentes para alcançar o tamanho amostral proposto, totalizando ao final 10 USF para a seleção dos participantes.

Na seleção da amostra, para compensar perdas foi adicionado ao tamanho amostral 10 participantes a mais em cada das dez USF, por meio de convite à participação no estudo. Assim, foram selecionados 400 usuários, para atingir o mínimo de $(\mathrm{n}=238)$.

Os critérios de inclusão no estudo foram: ser usuário adulto ( $\geq 20$ anos) cadastrado nas USF sorteadas; diagnóstico de DCNT (diabetes tipo 2 e/ou hipertensão arterial sistêmica) acompanhado pela Unidade; ter disponibilidade para comparecer a Unidade. Já os critérios para a exclusão foram: apresentar dor bucal ou abcesso no dia da entrevista; recusar o exame clínico bucal; possuir estado físico e psicológico (informados pela USF) que impedissem a realização dos exames e compreensão do questionário.

A participação dos usuários se deu por meio de convite, realizado por Agentes Comunitárias de Saúde (pessoalmente, via visitas domiciliares, comparecimento em consulta ou grupo HiperDia). As avaliações ocorreram durante o funcionamento das USF, em datas, horários e locais estabelecidos pelo próprio gestor.

\section{Coleta de Dados}

A coleta de dados foi realizada de julho a dezembro de 2019, por uma cirurgiã-dentista, treinada previamente, entre maio e junho de 2019, por uma examinadora "padrão-ouro", incluindo discussões teóricas e práticas por oito horas, a fim de obter pelo menos $90 \%$ de concordância para cárie, presença de biofilme dental visível e bolsa periodontal ${ }^{27,28}$. A concordância intraobservador variou de 90,6\% a 100\% para as condições dentárias e doença periodontal, estando dentro dos padrões de confiabilidade ${ }^{29}$.

Inicialmente foi realizada uma coleta piloto com usuários $(\mathrm{n}=18)$ em uma das USF sorteadas. Ao considerar que não houve necessidade de alterações no padrão de coleta e nem adaptação do questionário, os participantes do estudo piloto fizeram parte da amostra final.

A coleta de dados aconteceu nas dependências das USF, com aplicação de questionário, realização de exame clínico bucal e coleta em prontuários clínicos.

Foi aplicado um questionário, com 66 perguntas objetivas adaptadas ${ }^{30}$, para se obter os dados sobre os fatores sociodemográficos, comportamento e determinantes em saúde A 
LS foi avaliada utilizando o 14-Item Health Literacy Scale (HLS-14) ${ }^{31}$, traduzido e validado no Brasil por Batista et al ${ }^{19}$, com 14 questões em escala tipo Likert (5 pontos), com as categorias: "discordo muito", "discordo", "nem concordo nem discordo", "concordo" e "concordo muito"; e pontuação total variando entre 14 e 70 pontos, em que maiores pontuações indicam melhor LS. As questões de 1 a 5 são relacionadas à dimensão funcional e têm seu score invertido, ou seja, concordar com as afirmativas significa possuir baixa LS, e as demais questões relacionadas à literacia comunicativa (6 a 10) e crítica (11 a 14).

As condições clínicas avaliadas foram a presença de biofilme dental visível (em pelo menos uma superfície) de acordo com Ainamo e Bay $(1975)^{27}$, índice de dentes permanentes Cariados, Perdidos e Obturados (CPOD) e Índice Periodontal Comunitário (CPI) ${ }^{28}$. Os participantes foram examinados nas dependências das USF, sentados em cadeiras, com auxílio de luz natural, utilizando sonda tipo "Ball Point" e espelho bucal clínico, estéreis, segundo os critérios estabelecidos pela $\mathrm{OMS}^{28}$. Os dados clínicos referentes à diabetes e pressão arterial sistêmica mais recentes foram extraídos dos prontuários dos participantes nas USF.

\section{Variáveis de Estudo}

A LS foi considerada como variável explanatória (Figura 1), avaliada através do HLS-14 em que a soma do score varia entre 14 e 70 pontos, com pontuações mais altas indicando maior LS. A LS foi dividida, após análise descritiva, nos tercis em: baixa (0 a 33 pontos), média (de 34 a 46 pontos), e alta ( $>46$ pontos) ${ }^{34}$.

As variáveis foram selecionadas de acordo com o modelo conceitual teórico adaptado para o estudo (Figura 1), categorizadas em:

Exógenas: idade (contínua), sexo (mulher e homem) e cor da pele (branca e outras "amarela, preta ou parda");

Determinantes primários em saúde: renda pessoal (até um salário mínimo, e acima de um salário mínimo) e escolaridade (menos que 4 anos, ou até os 4 anos completos, e 5 anos ou mais - ensino fundamental 1 e 2), com inclusão de analfabetos e considerando o ensino fundamental como ponto de corte para a idade, desta amostra;

Determinantes intermediários em saúde: comportamentos relacionados à saúde, como escovação dental (3 ou mais vezes/dia, e até 2 vezes/dia), fio dental (usa diariamente, e não

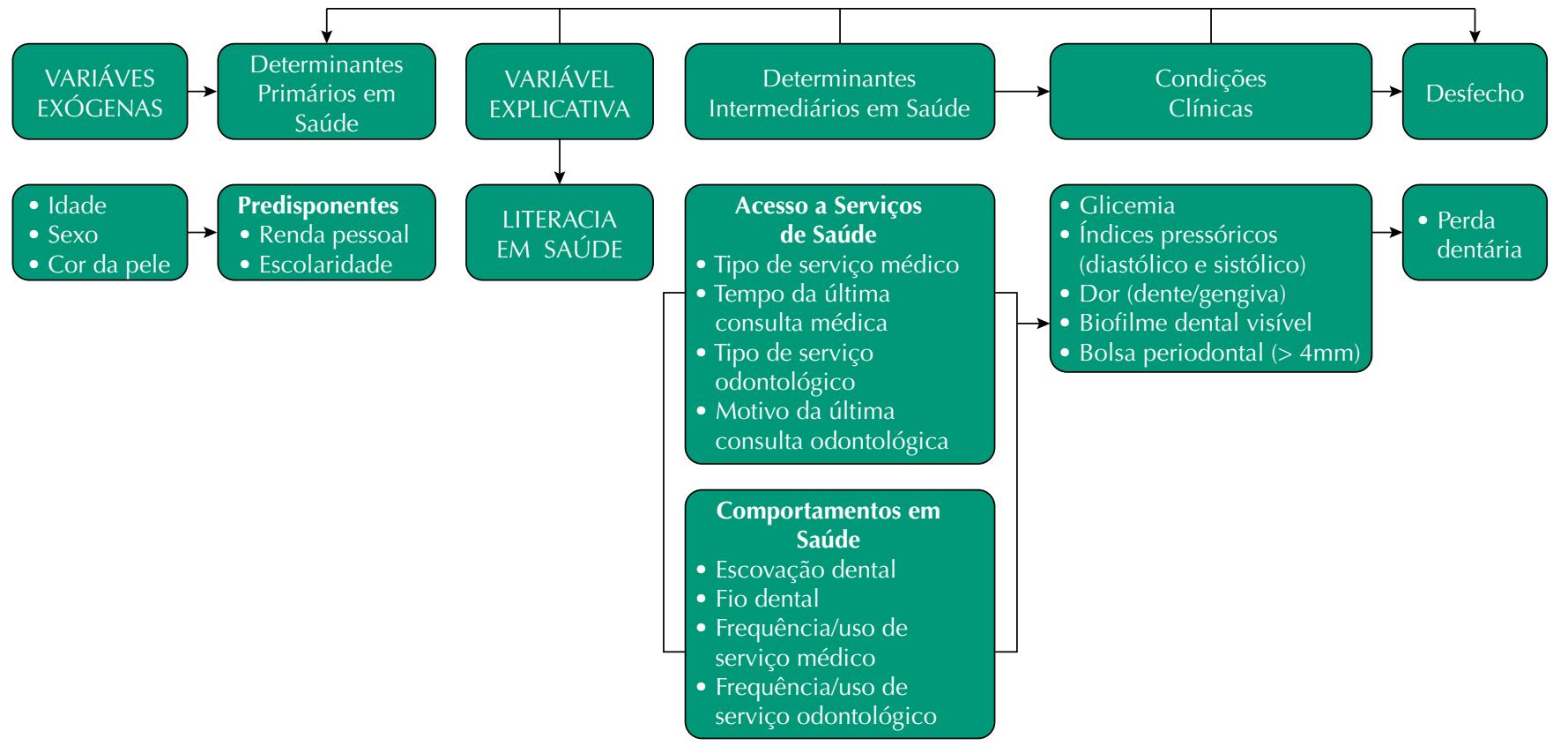

Figura 1. Modelo teórico conceitual para literacia em saúde associada às perdas dentárias. 
usa diariamente), frequência/uso de serviço médico e odontológico (uma vez ao ano ou mais, e menos que uma vez ao ano);

Acesso a serviços de saúde: tipo de serviço médico ou odontológico (público ou privado), tempo da última consulta médica (menos de um ano, e mais de um ano) e motivo da consulta odontológica (rotina, dor ou necessidade) adaptados ${ }^{30,33}$;

Condições clínicas: dor (dentes e/ou gengivas; sem dor, e alguma dor) adaptado ${ }^{30}$, biofilme dental visível (sim para pelo menos uma superfície com biofilme, ou não) e bolsa periodontal (CPI código 3 ou 4, bolsa $>4 \mathrm{~mm}$ ) (sim ou não) ${ }^{28,33}$. O controle glicêmico monitorado por glicemia de jejum (alterada $<126 \mathrm{mg} / \mathrm{dl}$ ), diabetes mellitus ( $127 \mathrm{mg} / \mathrm{dl}$ ou mais), hemoglobina glicada (HbAlc) (até 7,0\%, e 7,1\% ou mais) 34,35 , e HAS considerando normal (sistólica [< $130 \mathrm{mmHg}$ ] e diastólica [85-89]), e hipertensos com (sistólica [ $\geq 140 / 90 \mathrm{mmHg}]$ e diastólica [90-99mmHg ou mais]), ou em uso de medicação anti-hipertensiva ${ }^{36}$.

O desfecho do estudo (variável dependente) foi à perda dentária, cujo ponto de corte foi baseado na teoria do arco dentário reduzido, que considera satisfatória a presença de dez pares de dentes ocludentes sem lacunas estéticas. ${ }^{37} \mathrm{O}$ cálculo de dentes perdidos foi realizado pelo código 4 (dente perdido por cárie) e 5 (dente perdido por outros motivos) do CPOD, e a variável perda dentária foi categorizada em: tem 20 ou mais dentes presentes, tem entre 19 e 1 dentes, e edêntulos (nenhum dente presente). Os terceiros molares foram excluídos do exame, por isso a perda dentária total foi ter perdido 28 dentes.

Conforme ilustrado na Figura 1, foi construído para o estudo um modelo teórico conceitual de determinantes da saúde baseado em Nutbeam ${ }^{38}$, considerando a literacia em saúde, o modelo adaptado de Sørensen ${ }^{39}$ et al. e Martins ${ }^{40}$ et al., para análise das condições de saúde bucal sob os determinantes primários e os comportamentos de saúde ${ }^{41}$.

\section{Análise dos dados}

A análise foi realizada no programa Statistical Package for the Social Sciences (SPSS), versão 20.0. Primeiramente, realizaram-se as análises descritivas para obter a frequência, média, mediana, desvio-padrão e teste Qui-Quadrado das variáveis coletadas com base no modelo teórico (Figura 1), com nível de significância de 5\%.

Posteriormente foi feita a análise de modelos de regressão logística ordinal para a condição perdas dentárias (3 categorias). Realizou-se análises de regressão logística com abordagem hierárquica, segundo o modelo apresentado na Figura 1. Para a inclusão no modelo, em cada bloco foi considerado o corte de $\mathrm{p}<0,20$ e a significância no modelo final foi $\mathrm{p}<0,05$.

Os ajustes foram: Modelo 1: modelo de regressão com idade e escolaridade; Modelo 2: ajustado por tipo de serviço odontológico e LS; Modelo 3: ajuste para bolsa periodontal, dor dentes/gengivas, biofilme dental visível e glicemia; Modelo 4: ajustes dos Modelos 1 e 2 com escolaridade, LS e idade; Modelo 5: ajustes dos Modelos 1, 2 e 3,escolaridade, LS, idade, frequência/uso odontológico e uso de fio dental; Modelo 6: ajustes dos Modelos 1, 2, 4 e 5 com escolaridade, LS, idade, frequência/uso odontológico, uso de fio dental, bolsa periodontal, biofilme dental visível e glicemia.

\section{Aspectos Éticos}

O protocolo do estudo foi submetido e aprovado pelo Comitê de Ética em Pesquisa sob o CAAE 94104618.7.0000.5418. O Termo de Consentimento Livre e Esclarecido foi assinado previamente por todos os participantes.

\section{RESULTADOS}

Participaram desta pesquisa 238 usuários portadores das doenças crônicas bucais acompanhados em dez USF, sendo 7,2\% ( $\mathrm{n}=17)$ dos usuários tinham diabetes, 46,6\% ( $\mathrm{n}=111)$ 
Tabela 1. Características dos níveis de literacia em saúde e fatores associados com total, valor de p $(<0,05)$, em 238 indivíduos com doenças crônicas, usuários da Atenção Primária em Saúde em Piracicaba, SP, Brasil, 2019.

\begin{tabular}{|c|c|c|c|c|c|c|}
\hline \multirow{2}{*}{ Classificação das variáveis } & & \multicolumn{5}{|c|}{ Classificação da Literacia em Saúde (LSa) } \\
\hline & & $\begin{array}{c}\text { Total } \\
\text { n }(\%)\end{array}$ & $\begin{array}{c}\text { Baixa LS } \\
\text { n (\%) }\end{array}$ & $\begin{array}{c}\text { Média LS } \\
\text { n (\%) } \\
\end{array}$ & $\begin{array}{c}\text { Alta LS } \\
\text { n (\%) }\end{array}$ & $\mathbf{p}$ \\
\hline \multirow{2}{*}{\multicolumn{7}{|c|}{ Variáveis exógenas }} \\
\hline & & & & & & \\
\hline Idade média em anos (DP) & $62,7( \pm 10,55)$ & $238(100)$ & $66,1( \pm 8,66)$ & $62,8( \pm 9,78)$ & $58,2( \pm 12,00)$ & $<0,001$ \\
\hline \multirow{2}{*}{ Sexo } & Mulher & $165(69,3)$ & $62(37,6)$ & $56(33,9)$ & $47(28,5)$ & \multirow{2}{*}{0,518} \\
\hline & Homem & $73(30,7)$ & $22(30,1)$ & $29(39,8)$ & $22(30,1)$ & \\
\hline \multirow{2}{*}{ Cor da pele } & Branca & $168(80,0)$ & $59(35,1)$ & $57(33,9)$ & $52(31,0)$ & \multirow{2}{*}{0,322} \\
\hline & Outra & $42(20,0)$ & $14(33,3)$ & $19(45,2)$ & $9(21,4)$ & \\
\hline \multicolumn{7}{|l|}{ Determinantes primários em saúde } \\
\hline \multirow{2}{*}{ Renda Pessoal } & Acima de $1^{\text {(b) }} \mathrm{SM}$ & $165(69,3)$ & $57(34,5)$ & $56(33,9)$ & $52(31,5)$ & \multirow{2}{*}{0,419} \\
\hline & Até $1{ }^{\text {(b) }} \mathrm{SM}$ & $73(30,7)$ & $27(37,0)$ & $29(39,7)$ & $17(23,3)$ & \\
\hline \multirow{3}{*}{ Escolaridade } & Menos que 4 anos & $86(36,1)$ & $38(44,2)$ & $31(36,0)$ & $17(19,8)$ & \multirow{3}{*}{$<0,001$} \\
\hline & Até 4 anos completos & $101(42,4)$ & $38(37,6)$ & $40(39,6)$ & $23(22,8)$ & \\
\hline & 5 anos ou mais & $51(21,4)$ & $08(15,7)$ & $14(27,5)$ & $29(56,9)$ & \\
\hline \multicolumn{7}{|l|}{ Determinantes intermediários em saúde } \\
\hline \multicolumn{7}{|l|}{ Acesso a serviços de saúde } \\
\hline \multirow{2}{*}{ Tipo de serviço médico } & Público & $212(89,5)$ & $80(37,7)$ & $75(35,4)$ & $57(26,9)$ & 0067 \\
\hline & Privado & $25(10,5)$ & $04(16,0)$ & $10(40,0)$ & $11(44,0)$ & $0,00 \%$ \\
\hline Tipo de servico odontológico & Público & $115(48,7)$ & $32(27,8)$ & $52(45,2)$ & $31(27,0)$ & 0.010 \\
\hline 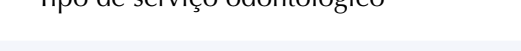 & Privado & $121(51,3)$ & $52(43,0)$ & $33(27,3)$ & $36(29,8)$ & 0,010 \\
\hline Motivo da consulta odontológica & Rotina & $145(61,2)$ & $52(35,9)$ & $54(37,2)$ & $39(26,9)$ & 0.728 \\
\hline 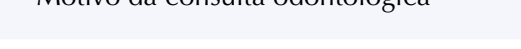 & Dor ou necessidade & $92(38,8)$ & $32(34,8)$ & $31(33,7)$ & $29(31,5)$ & \\
\hline Tempo da última consulta médica & Até 1 ano & $218(91,6)$ & $80(36,7)$ & $75(34,4)$ & $63(28,9)$ & 0.259 \\
\hline tempo ua uituma consurla meured & Mais de 1 ano & $20(8,4)$ & $04(20,0)$ & $10(50,0)$ & $06(30,0)$ & 0,239 \\
\hline Comportamentos em saúde & & & & & & \\
\hline Fscovacão & Até 2 vezes/dia & $103(43,3)$ & $47(45,6)$ & $29(28,2)$ & $27(26,2)$ & 0,012 \\
\hline Lscuvą̧au & 3 ou mais vezes/dia & $135(56,7)$ & $37(27,4)$ & $56(41,5)$ & $42(31,1)$ & \\
\hline Fio dental & Usa diariamente & $76(31,9)$ & $11(14,5)$ & $32(42,1)$ & $33(43,4)$ & $<0,001$ \\
\hline Tiv netral & Não usa diariamente & $162(68,1)$ & $73(45,1)$ & $53(32,7)$ & $36(22,2)$ & \\
\hline Frequência/uso de servico médico & 1 vez/ano ou mais (uso regular) & $181(76,1)$ & $70(38,7)$ & $66(36,5)$ & $45(24,9)$ & 0.030 \\
\hline 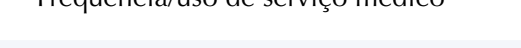 & -1 vez/ano (uso irregular) & $57(23,9)$ & $14(24,6)$ & $19(33,3)$ & $24(42,1)$ & \\
\hline Frequência/uso de servico odontológico & +1 vez/ano ou mais (uso regular) & $58(25,2)$ & $08(13,8)$ & $24(41,4)$ & $26(44,8)$ & 0.001 - \\
\hline riequencia/uso de serviço oaontologico & 1 vez/ano (uso irregular) & $172(74,8)$ & $75(46,3)$ & $59(34,3)$ & $38(22,1)$ & $<0,001$ \\
\hline Condições clínicas & & & & & & \\
\hline & Tem até 20 dentes & $75(31,5)$ & $15(20,0)$ & $24(32,0)$ & $36(48,0)$ & $<0,001$ \\
\hline Perda dentária & Tem 1 a 19 dentes & $86(36,1)$ & $33(38,4)$ & $32(37,2)$ & $21(24,4)$ & \\
\hline & Edêntulo & $77(32,4)$ & $36(46,8)$ & $29(37,7)$ & $12(15,6)$ & \\
\hline Dor (dentes/gengivas) & Sem dor & $156(66,5)$ & $57(36,5)$ & $47(30,1)$ & $52(33,3)$ & 0.029 \\
\hline Dor (uentes/gengivas) & Alguma dor & $82(34,5)$ & $27(32,9)$ & $38(46,3)$ & $17(20,7)$ & 0,020 \\
\hline Glicemia & Até 126 dmgl & $113(47,5)$ & $41(36,3)$ & $37(32,7)$ & $35(31,0)$ & 0,693 \\
\hline & 127 dmgl ou mais & $125(52,5)$ & $43(43,4)$ & $48(38,4)$ & $34(27,2)$ & \\
\hline Hemoglobina Glicada (HbA1c) & Até $7,0 \%$ & $92(38,7)$ & $35(38,0)$ & $30(32,6)$ & $27(29,3)$ & 0.643 \\
\hline 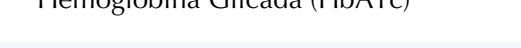 & $7,1 \%$ ou mais & $146(61,3)$ & $49(33,6)$ & $55(37,7)$ & $42(28,8)$ & 0,043 \\
\hline Pressão Arterial Sistólica & Até 139 mmhg & $174(73,1)$ & $69(39,7)$ & $61(35,1)$ & $44(25,3)$ & 0.036 \\
\hline न & 140 mmhg ou mais & $64(26,9)$ & $15(23,4)$ & $24(37,5)$ & $25(39,1)$ & \\
\hline Pressão Arterial Diastólica & Até 89 mmhg & $210(88,2)$ & $77(36,7)$ & $70(33,3)$ & $63(30,0)$ & 0.110 \\
\hline Pressao Amerial Diastomica & $90 \mathrm{mmhg}$ ou mais & $28(11,8)$ & $07(25,0)$ & $15(53,6)$ & $06(21,4)$ & 0,110 \\
\hline Bolsa periodontal (> 4mm) & Sim & $137(57,6)$ & $57(41,6)$ & $39(35,8)$ & $31(22,6)$ & 0.017 \\
\hline 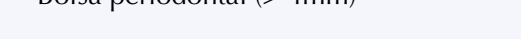 & Não & $101(42,4)$ & $27(26,7)$ & $36(35,6)$ & $38(37,6)$ & 0,010 \\
\hline Biofilme dental visí & Sim & $81(34,0)$ & $20(24,7)$ & $30(37,0)$ & $31(38,3)$ & 0,021 \\
\hline Diominte cental visivel & Não & $155(65,1)$ & $63(40,6)$ & $55(35,5)$ & $37(23,9)$ & \\
\hline
\end{tabular}

Fonte: elaborada pelos autores (2020). Teste do Qui-quadrado.

a Literacia em Saúde (LS) tricotomizada em 3 grupos, 1. (acima de 46 pontos) classificado em alta literacia, 2. (de 34 a 46 pontos), média, e 3 . (0 a

38 pontos) baixa literacia. HLS-14 (Suka et al., 2013; Batista et al., 2020).

b Salário mínimo (SM) brasileiro = R\$998.00 (Dez / 2019). 3. Para perda dentária foram excluídos os terceiros molares considerando o total de 28 dentes. 
deles tinham HAS, e 46,2\% ( $n$ = 110) apresentavam diabetes e HAS. Dois usuários se recusaram a realizar o exame clínico e houve uma perda amostral, devido ao não comparecimento, de 162 usuários. A taxa de resposta foi 59,5\%, porém, atingiu-se o mínimo pretendido.

A média de idade dos participantes foi de $62,7( \pm 10,55)$ anos, com maioria de mulheres, $69,3 \%(n=165)$. Apresentaram menor escolaridade 78,5\% ( $n=187)$ dos participantes e o nível de baixa LS ocorreu em 33,8\% ( $\mathrm{n}=84)$, o de média em $36,8 \%(\mathrm{n}=85)$ e alta em $29,3 \%(n=69)$.

A baixa LS foi associada a menor frequência de escovação, uso irregular de serviço odontológico, uso irregular de fio dental, maior prevalência de edentulismo, presença de alguma dor (gengiva/dentes), pressão arterial sistólica, bolsa periodontal ( $>4 \mathrm{~mm}$ ) e biofilme dental visível (Tabela 1).

A média de dentes perdidos na amostra foi de 14,63 ( \pm 9,36). Na Figura 2 pode-se observar os gradientes de perdas dentárias em relação aos gradientes de literacia em saúde, quanto maior o gradiente de literacia, menor a prevalência de edentulismo, e maior prevalência da presença de 20 ou mais dentes.

A perda dentária esteve associada à literacia no Modelo 2 pela média LS (OR = 2,80; IC95\% 1,50-5,20) e baixa LS (OR = 4,70; IC95\% 2,50-8,82), quando ajustadas por tipo de serviço odontológico. Porém, a LS perdeu a significância quando foram incluídas outras variáveis a partir do Modelo 3, ajustado por bolsa periodontal visível, dor (dentes/gengivas), biofilme dental visível e glicemia (Tabela 2).

No modelo final, as perdas dentárias foram associadas à maior idade (OR =1,12; IC95\% 1,07-1,17), menor escolaridade (OR = 3,43; IC95\% 1,17-10,10), índices mais altos de glicemia (OR = 1,98; IC95\% 1,00-3,92), não uso de fio dental ( $\mathrm{OR}=4,58$; IC95\% 1,75-7,31), uso irregular do serviço odontológico (OR = 2,60; IC95\% 1,32-5,12), presença de bolsa periodontal > 4mm (OR = 0,31; IC95\% 0,01-0,08) e biofilme dental visível (OR = 7,23; IC95\%3,19-16,41) (Tabela 3).

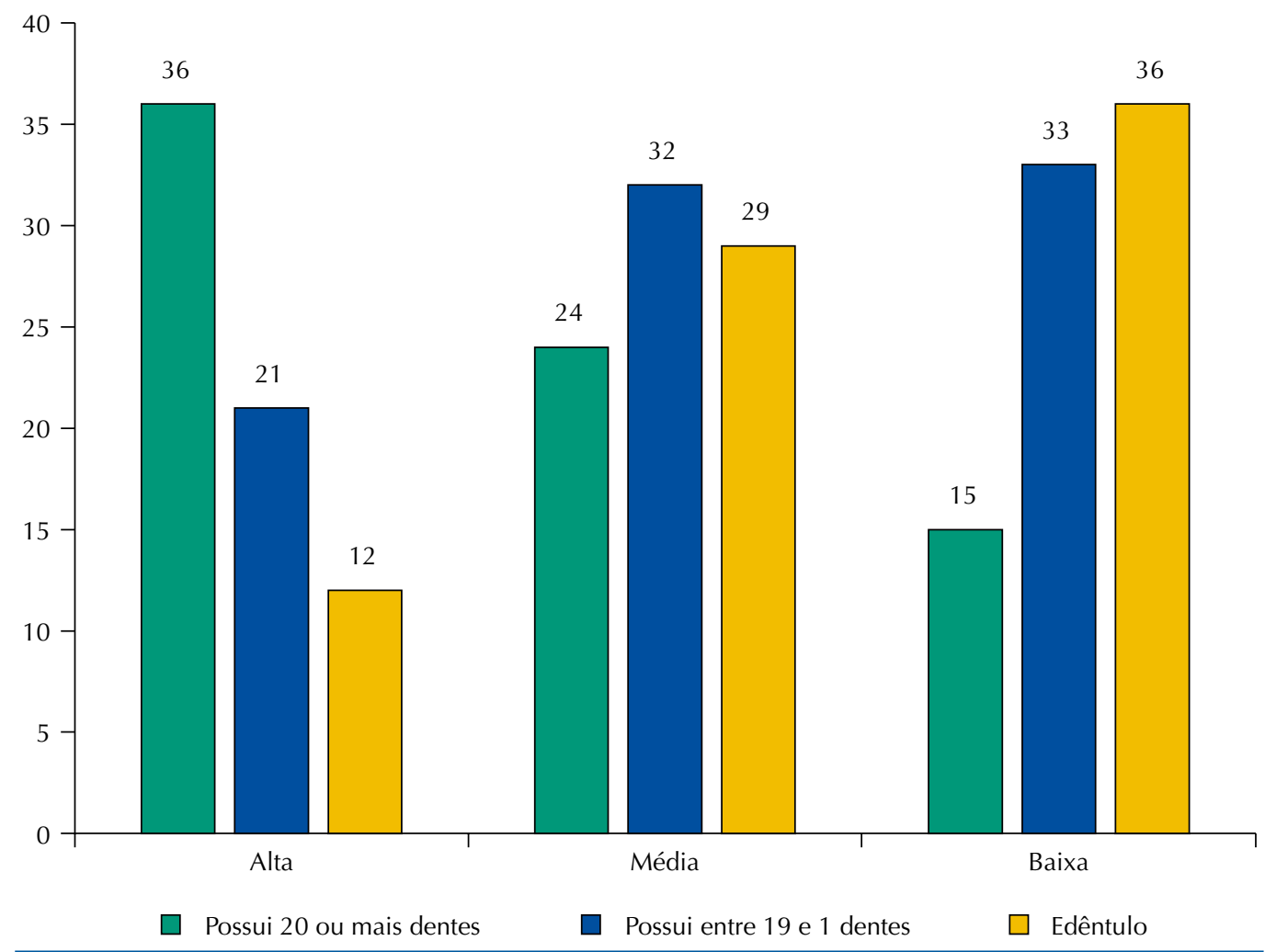

Figura 2. Gradientes da perda dentária de acordo com os gradientes de literacia em saúde. 
Tabela 2. Características das perdas dentárias e variáveis sociodemográficas acesso, comportamento e condições de saúde entre indivíduos ( $n=238)$ usuários portadores de doenças crônicas não transmissíveis, usuários da Atenção Primária em Saúde em Piracicaba, SP, Brasil, 2019.

\begin{tabular}{|c|c|c|c|c|c|c|c|}
\hline \multirow[b]{2}{*}{ Classificação das variáveis } & & \multicolumn{6}{|c|}{ Classificação das Perdas Dentáriasa } \\
\hline & & $\begin{array}{c}\text { Tem } 20 \text { dentes } \\
\text { ou mais }\end{array}$ & $\begin{array}{c}\text { Tem } \\
\text { entre } 1 \text { e } \\
19 \text { dentes }\end{array}$ & Edêntulos & $\begin{array}{c}\text { OR } \\
\text { Bruto }\end{array}$ & IC95\% & $\mathbf{p}$ \\
\hline \multicolumn{8}{|l|}{ Variáveis exógenas } \\
\hline Média em anos (DP) & $62,7( \pm 10,55)$ & $54,07( \pm 9,03)$ & $64,63( \pm 8,24)$ & $68,83( \pm 7,84)$ & 1,38 & $1,10-1,17$ & $<0,001$ \\
\hline \multirow{2}{*}{ Sexo } & Mulher & $55(33,3)$ & $58(35,2)$ & $52(31,5)$ & 1 & & 0,444 \\
\hline & Homem & $20(27,4)$ & $28(38,4)$ & $25(34,2)$ & 1,22 & $0,74-2,02$ & \\
\hline \multirow{2}{*}{ Cor da pele } & Branca & $52(31,0)$ & $58(34,5)$ & $58(34,5)$ & 1 & & \\
\hline & Outra & $12(28,6)$ & $16(38,1)$ & $14(33,3)$ & 1,03 & $0,55-1,90$ & 0,938 \\
\hline \multicolumn{8}{|c|}{ Determinantes primários em saúde } \\
\hline \multirow{2}{*}{ Renda Pessoal } & Acima de $1^{\text {(b) }} \mathrm{SM}$ & $63(38,2)$ & $55(33,3)$ & $47(28,5)$ & 1 & & 0,003 \\
\hline & Até $1{ }^{(b)} S M$ & $12(16,4)$ & $31(42,5)$ & $30(41,1)$ & 2,18 & $1,31-3,64$ & \\
\hline \multirow{3}{*}{ Escolaridade } & Menos que 4 anos & $12(14,0)$ & $30(34,9)$ & $44(51,2)$ & 16,22 & $7,57-34,78$ & $<0,001$ \\
\hline & Até 4 anos completos & $27(26,7)$ & $43(42,6)$ & $31(30,7)$ & 6,88 & $3,37-14,06$ & \\
\hline & 5 anos ou mais & $36(70,6)$ & $13(25,5)$ & $2(3,9)$ & 1 & & \\
\hline \multirow{3}{*}{ Literacia em Saúde } & Alta & $36(52,2)$ & $21(30,4)$ & $12(17,4)$ & 4,26 & $2,91-6,23$ & $<0,001$ \\
\hline & Média & $24(28,2)$ & $32(37,6)$ & $29(34,1)$ & 2,73 & $1,88-3,97$ & $<0,001$ \\
\hline & Baixa & $15(17,9)$ & $33(39,3)$ & $36(42,9)$ & 1 & & \\
\hline
\end{tabular}

\section{Determinantes intermediários em saúde}

\begin{tabular}{|c|c|c|c|c|c|c|c|}
\hline \multicolumn{8}{|l|}{ Acesso a serviços de saúde } \\
\hline \multirow{2}{*}{ Tipo de serviço médico } & Público & $62(29,2)$ & $67(36,3)$ & $73(34,4)$ & 2,37 & $1,09-5,16$ & 0,029 \\
\hline & Privado & $12(48,0)$ & $9(36,0)$ & $4(16,0)$ & 1 & & \\
\hline \multirow{2}{*}{ Tipo de serviço odontológico } & Público & $30(26,1)$ & $48(41,7)$ & $37(32,2)$ & 1,29 & $0,81-2,06$ & 0,291 \\
\hline & Privado & $35(47,2)$ & $37(30,6)$ & $39(32,2)$ & 1 & & \\
\hline \multirow{2}{*}{ Motivo da consulta odontológica } & Rotina & $46(31,7)$ & $47(32,4)$ & $52(35,9)$ & 1 & & \\
\hline & Dor ou necessidade & $29(31,5)$ & $39(42,4)$ & $24(26,1)$ & 0,80 & $0,50-1,30$ & 0,300 \\
\hline \multirow{2}{*}{ Tempo da última consulta médica } & Menos de 1 ano & $71(32,6)$ & $77(35,3)$ & $70(32,7)$ & 1 & & \\
\hline & 1 vez ao ano ou mais & $4(20,0)$ & $9(45,0)$ & $7(35,0)$ & 3,39 & $2,04-5,63$ & $<0,001$ \\
\hline \multicolumn{8}{|l|}{ Comportamentos em saúde } \\
\hline \multirow{2}{*}{ Escovação } & Até 2 vezes/dia & $21(20,4)$ & $34(33,0)$ & $48(4,66)$ & 2,91 & $1,78-4,76$ & $<0,001$ \\
\hline & 3 ou mais vezes/dia & $54(40,0)$ & $52(38,5)$ & $29(21,5)$ & 1 & & \\
\hline \multirow{2}{*}{ Fio dental } & Usa diariamente & $46(60,5)$ & $28(36,8)$ & $0(0,0)$ & 1 & & \\
\hline & Não usa diariamente & $29(17,9)$ & $58(35,8)$ & $75(43,3)$ & 9,16 & $5,13-16,37$ & $<0,001$ \\
\hline \multirow{2}{*}{ Frequência/uso de serviço médico } & 1 vez/ano ou mais (uso regular) & $57(31,5)$ & $63(34,8)$ & $61(33,7)$ & 1 & & \\
\hline & -1 vez/ano (uso irregular) & $18(31,6)$ & $23(40,4)$ & $16(28,1)$ & 0,88 & $0,58-1,33$ & 0,537 \\
\hline \multirow{2}{*}{ Frequência/uso de serviço odontológico } & 1 vez/ano ou mais (uso regular) & $34(58,6)$ & $21(36,2)$ & $3(5,2)$ & 1 & & \\
\hline & -1 vez/ano (uso irregular) & $39(22,7)$ & $63(36,6)$ & $70(40,7)$ & 5,77 & $1,95-17,10$ & $\mathbf{0 , 0 0 2}$ \\
\hline Condições clínicas & Sem dor & $45(28,8)$ & $53(34,0)$ & $58(37,2)$ & 1 & & \\
\hline Dor (dentes/gengivas) & Alguma dor & $30(36,6)$ & $33(40,2)$ & $19(23,2)$ & 0,61 & $0,37-1,01$ & 0,054 \\
\hline \multirow{2}{*}{ Glicemia } & Até 126 dmgl & $38(33,6)$ & $40(35,4)$ & $35(31,0)$ & 1 & & \\
\hline & $127 \mathrm{dmgl}$ ou mais & $37(29,6)$ & $46(36,8)$ & $42(33,6)$ & 1,17 & $1,05-1,30$ & 0,006 \\
\hline \multirow{2}{*}{ Hemoglobina Glicada (HbA1c) } & Até $7,0 \%$ & $35(38,0)$ & $27(29,3)$ & $30(32,6)$ & 1 & & \\
\hline & $7,1 \%$ ou mais & $40(27,4)$ & $59(40,4)$ & $47(32,2)$ & 1,27 & $0,55-2,95$ & 0,579 \\
\hline \multirow{2}{*}{ Pressão Arterial Sistólica } & Até 139 mmhg & $55(31,6)$ & $64(36,8)$ & $55(31,6)$ & 1 & & \\
\hline & 140 mmhg ou mais & $20(31,2)$ & $22(34,4)$ & $22(34,4)$ & 1,08 & $0,90-1,29$ & 0,424 \\
\hline \multirow{2}{*}{ Pressão Arterial Diastólica } & Até 89 mmhg & $65(31,0)$ & $80(38,1)$ & $65(31,0)$ & 1 & & \\
\hline & $90 \mathrm{mmhg}$ ou mais & $10(35,7)$ & $6(21,4)$ & $12(42,9)$ & 1,20 & $0,31-4,63$ & 0,789 \\
\hline \multirow{2}{*}{ Bolsa periodontal $(>4 \mathrm{~mm})$} & Sim & $62(61,4)$ & $39(38,6)$ & $0(0,0)$ & 0,39 & $0,01-0,47$ & 0,039 \\
\hline & Não & $13(9,5)$ & $47(34,3)$ & $77(56,2)$ & 1 & & \\
\hline \multirow{2}{*}{ Biofilme dental visível } & Sim & $39(48,1)$ & $42(51,9)$ & $0(0,0)$ & 6,25 & $0,26-148,10$ & 0,256 \\
\hline & Não & $34(21,9)$ & $44(28,4)$ & $77(49,7)$ & 1 & & \\
\hline
\end{tabular}

Fonte: Elaboração dos autores (2020).

a Teoria do Arco Reduzido (Armellini e Fraunhofer, 2004).

b Salário mínimo (SM) brasileiro = R\$998.00 (Dez / 2019). 


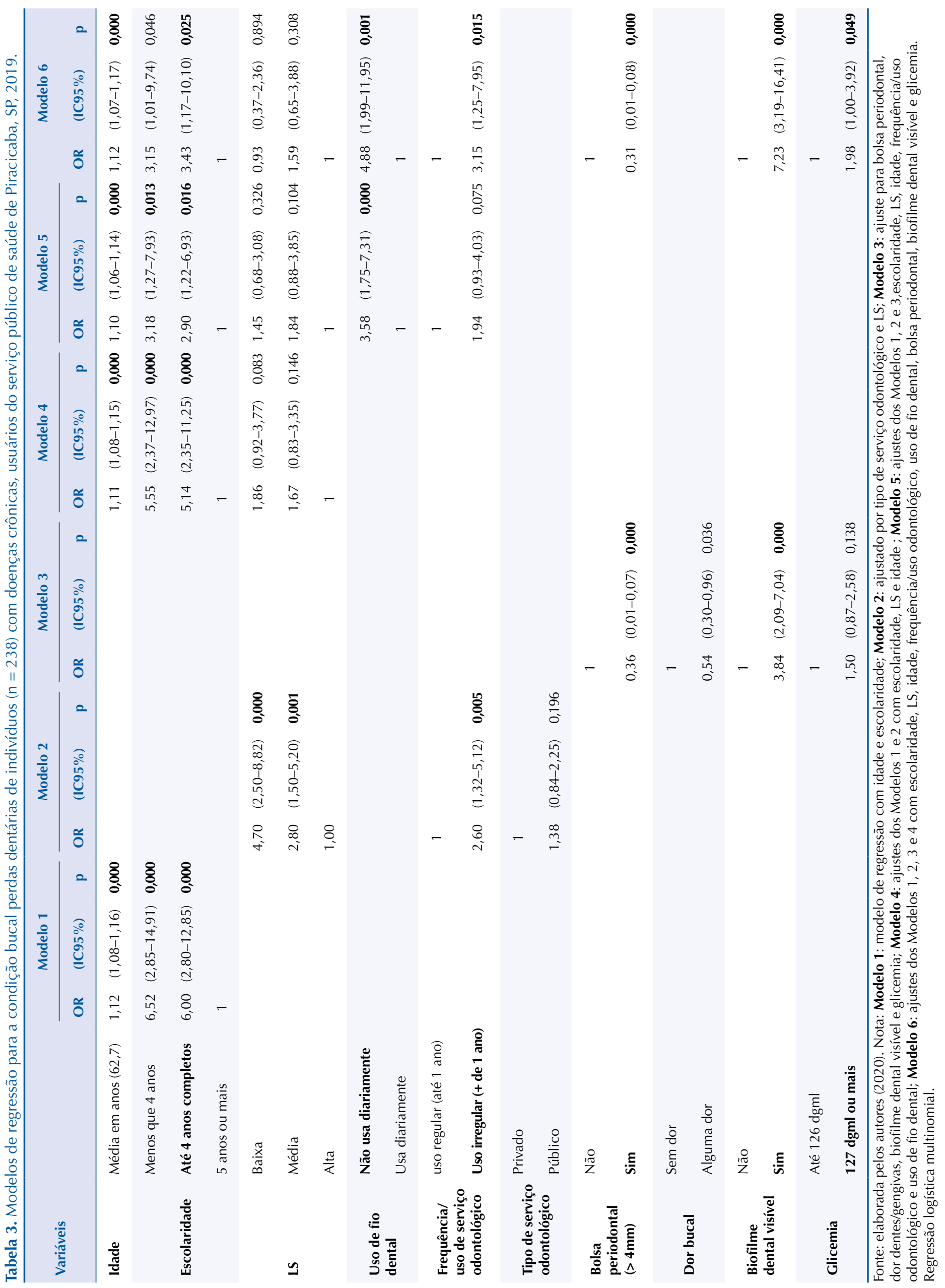




\section{DISCUSSÃO}

A LS foi fator associado significativo para a perda dentária, mesmo com os determinantes intermediários em saúde, acesso ao serviço e comportamentos em saúde, mas, quando ajustada por fatores sociodemográficos e condições clínicas, perdeu significância. Observou-se que conforme aumentam os gradientes de literacia em saúde, diminui-se o edentulismo. Porém, sabe-se que perdas dentárias sofrem influência de múltiplos fatores e, por isso, como demonstrado no modelo final desta análise, foi associada à idade, à escolaridade, ao não uso de fio dental, ao uso irregular de serviço odontológico, à presença de bolsa periodontal, ao biofilme dental visível e ao índice glicêmico. Destaca-se que, esses mesmos fatores, à exceção do índice glicêmico, também foram os fatores associados à LS, na análise bivariada.

A LS tem sido considerada um fator intermediário determinante nos comportamentos e desfechos em saúde ${ }^{17} \mathrm{e}$ fundamental nos dias atuais para compreensão das informações em saúde, o que acarreta em manutenção e recuperação da saúde ${ }^{15}$, conforme demonstram estudos recentes ${ }^{18,42}$. A associação com fatores intermediários de comportamento, como o uso regular de fio dental, o acesso ao serviço, a frequência e o tipo de serviço odontológico, também tem sido observada na literatura ${ }^{11,43,44}$, ratificando o papel da LS na tomada de decisão em saúde. Sendo assim, fica evidente que a LS é um determinante social importante a ser considerado como estratégia para se trabalhar na promoção da saúde e do bem-estar.

O uso de ajuste de modelos deste estudo pôde indicar que o principal desfecho em saúde bucal, a perda dentária, deriva de diversos fatores de risco acumulados ao longo da história do indivíduo e, por isso, os comportamentos de saúde, escovação dental, uso de fio dental e as condições clínicas, como as doenças bucais e gerais, condições mais proximais, acabam reduzindo o impacto da LS neste desfecho, quando esses aspectos são considerados. Porém observa-se que, conforme o aumento do gradiente literacia em saúde, aumenta também o edentulismo.

Nesse sentido, faz-se necessário demonstrar o papel intermediário da LS na redução de perdas dentárias, que é o principal resultado de saúde bucal, considerada um desafio global, e em doenças periodontais que, associadas às DCNT, têm consequências graves e impactam na qualidade de vida da pessoa ${ }^{9}$, embora ainda seja inconclusiva a associação entre perdas dentárias e a LS na literatura ${ }^{18,45}$.

Neste estudo, as perdas dentárias demonstraram associações com maior idade devido a um efeito de corte, ou seja, relacionado ao resultado histórico das políticas públicas em saúde bucal no Brasil e seu impacto na população ${ }^{14}$. A escolaridade, outro determinante primário em saúde, também foi associada às perdas dentárias, corroborando outros achados, em que menor escolaridade é associada a maior prevalência de perdas dentárias ${ }^{46,47}$, e estabelecida como indicador de risco em saúde ${ }^{48}$. Fatores sociodemográficos, econômicos e etários, encontrados também em outros estudos, são fatores estruturais e, portanto, difíceis de serem modificados ${ }^{49,50}$. A LS é um fator modificável e está fortemente associada a comportamentos e aspectos associados às perdas dentárias, embora sejam necessários outros estudos para esclarecer a associação com desfechos em saúde. A OMS destaca que a LS é peça chave para o desenvolvimento da promoção de saúde ${ }^{15}$, sendo também importante indicador de disparidades sociais ${ }^{15}$.

A variável renda foi associada à perda dentária na análise univariada, porém, no ajuste com escolaridade e idade, perde a significância (não foi associada à LS). Isso pode ter ocorrido devido à colinearidade com escolaridade e/ou à homogeneidade da amostra no aspecto socioeconômico onde a maioria tem renda de um salário mínimo ou mais e estudou até quatro anos.

Nesta amostra, foram encontradas associações de perdas dentárias com os comportamentos, como uso irregular de fio dental e uso de serviço odontológico, variáveis que impactam no aumento da prevalência da perda dentária em indivíduos com doenças crônicas, de acordo com estudo realizado em Santa Rita, Paraíba, Brasil ${ }^{51}$. Em relação às condições clínicas presença de biofilme dental e bolsa periodontal, também foram encontradas associações 
em demais estudos, reforçando a influência das DCNT sobre a doença periodontal, perda dentária e consequências sistêmicas ${ }^{52,47}$. Neste estudo, apresentaram maior presença de bolsa periodontal os que possuíam mais dentes presentes na boca, levando em consideração o grande número de edêntulos.

Os comportamentos são influenciados pelos determinantes e impactam nas doenças crônicas, por isso, dar atenção aos tipos de determinantes de iniquidades ${ }^{53}$ pode determinar melhorias em saúde e nesse caso, a LS pode ser um agente modificador, empoderando o indivíduo e colocando-o como protagonista de sua saúde. Em suma, entender a LS associada a comportamentos em saúde torna-se um considerável preditor.

A associação entre alta glicemia e perdas dentárias, apontada neste estudo, comprova que a perda dentária é um indicador importante de saúde sistêmica, como também apontaram outros trabalhos ${ }^{5,55}$, sendo inclusive considerada como fator de risco para doenças cardíacas ${ }^{12,56}$ e para artrite reumatoide ${ }^{57}$. Além de potencializar condições mais graves de saúde, esta condição pode ser preditora de mortalidade ${ }^{58}$, fato de extrema relevância e que reforça a importância da abordagem da integralidade na saúde ${ }^{59}$.

Uma limitação deste estudo foi o recorte transversal, que impossibilitou uma inferência causal. Outro aspecto a ser considerado foi à homogeneidade da amostra, na qual todos os indivíduos apresentam doenças crônicas e possibilidade de comorbidades. Porém, a amostra é representativa dessa parcela da população que precisa ser estudada e inserida no serviço de saúde com olhar diferenciado e com maior consideração à saúde integral, já que é a faixa etária sobre a qual ocorre maior severidade de doenças bucais, principalmente a perda dentária ${ }^{60}$, seus determinantes podem causar reflexos na saúde sistêmica, portanto necessitando de rápida atenção.

Conhecer e identificar fatores de risco associados pode representar melhor manejo nos cuidados em saúde dessa população, bem como o desenvolvimento de estratégias de promoção da saúde. Uma vez que muitos sistemas de saúde no mundo não acompanham o aumento da carga de DCNT e as necessidades de saúde associadas da população ${ }^{61}$, este estudo mostra-se um relevante alerta para que gestores considerem a integralidade em saúde, bem como a LS, no trabalho de equipe multiprofissional ${ }^{62} \mathrm{e}$ em suas ações de políticas públicas voltadas a essa população. Para tanto, também foi mostrado o uso de uma ferramenta de mensuração da LS de fácil aplicação para equipes de saúde atuarem no controle das DCNT.

Quando mensurável, por meio de instrumentos usados por profissionais de saúde (como o utilizado neste estudo), a LS também será importante para a modificação dos desfechos clínicos, podendo ser modificada por meio de intervenções e ações de promoção de saúde, aumentando a autonomia das pessoas nas tomadas de decisão. Com isso, é relevante considerar a integralidade em saúde e a LS nas políticas de promoção da saúde.

\section{CONCLUSÃO}

A perda dentária esteve associada à literacia em saúde, quando ajustada por comportamentos em saúde, a partir da inclusão das variáveis sociodemográficas e condições clínicas, a literacia em saúde perdeu a significância. No modelo final, comportamentos, determinantes em saúde e condições clínicas foram indicadores de risco da perda dentária, demonstrando a multifatorialidade envolvida nesse fenômeno. Assim, sugere-se futuros estudos para compreender a perda dentária que abordem a literacia em saúde e integralidade.

\section{REFERÊNCIAS}

1. Malta DC, Andrade SSCA, Oliveira TP, Moura L, Prado RR, Souza MFM. Probabilidade de morte prematura por doenças crônicas não transmissíveis, Brasil e regiões, projeções para 2025. Rev Bras Epidemiol. 2019;22:E190030. https://doi.org/10.1590/1980-549720190030 
2. GBD 2019 Risk Factors Collaborators. Global burden of 87 risk factors in 204 countries and territories, 1990-2019: a systematic analysis for the Global Burden of Disease Study 2019. Lancet. 2020;396(10258):1223-49. https://doi.org/10.1016/S0140-6736(20)30752-2

3. Institute for Health Metrics and Evaluation. GBD 2019 Cause and Risk Summaries. Diabetes mellitus - Level 3 cause. Seattle, WA: University of Washington, IHME; c2020 [citado 6 nov 2020]. Disponível em: http://www.healthdata.org/results/gbd_summaries/2019/diabetes-mellitus-level-3-cause

4. Organização Pan-Americana da Saúde. COVID-19 afeta funcionamento de serviços de saúde para doenças crônicas não transmissíveis nas Américas. Brasília, DF: OPAS Brasil; 2020. [citado 3 out 2020]. Disponível em: https://www.paho.org/pt/noticias/17-6-2020-covid-19-afeta-funcion amento-servicos-saude-para-doencas-cronicas-nao

5. World Health Organization. Noncommunicable Diseases (NCD) Country Profiles 2018. Geneva (CH): WHO; 2018 [citado 29 out 2020]. Disponível em: https://apps.who.int/iris/handle/10665/274512

6. Secretaria de Estado da Saúde de São Paulo, Coordenadoria de Controle de Doenças, Centro de Vigilância Epidemiológica, Divisãode Doenças Crônicas Não Transmissíveis. Painel Mortalidade DCNT Estado de São Paulo 1980-2018. São Paulo: CVE; 2019 [citado 16 mar 2020]. Disponível em: https://docs.bvsalud.org/biblioref/2020/08/972096/dcnt_painel_esp_19802018.pdf

7. Hirschman KB, Shaid E, McCauley K, Pauly MV, Naylor MD. Continuity of care: the Transitional Care Model. Online J Issues Nurs. 2015;20(3):1. https://doi.org/10.3912/OJIN.Vol20No03Man01

8. Malta DC, Bernal RTI, Lima MG, Araújo SSC, Silva MMA, Freitas MIF, et al. Noncommunicable diseases and the use of health services: analysis of the National Health Survey in Brazil. Rev Saude Pública. 2017;51 Suppl 1:4s. https://doi.org/10.1590/s1518-8787.2017051000090

9. Peres MA, Macpherson LMD, Weyant RJ, Daly B, Venturelli R, Mathur MR, et al. Oral diseases: a global public health challenge. Lancet. 2019;394(10194):249-60. https://doi.org/10.1016/S0140-6736(19)31146-8

10. Institute for Health Metrics and Evaluation. GBD 2019 Cause and Risk Summaries. Edentulism and severe tooth loss - Level 4 cause. Seattle, WA: University of Washington, IHME; c2020 [acesso 6 nov 2020]. Disponível em: http://www.healthdata.org/results/gbd_ summaries/2019/edentulism-and-severe-tooth-loss-level-4-cause

11. Silva Junior MF, Batista MJ, Sousa MLR. Risk factors for tooth loss in adults: a population-based prospective cohort study. PLoS One. 2019;14(7):e0226794. https://doi.org/10.1371/journal.pone.0219240

12. Tsakos G, Quiñonez C. A sober look at the links between oral and general health [editorial]. J Epidemiol Community Health. 2013;67(5):381-2. https://doi.org/10.1136/jech-2013-202481

13. Dennis S, Williams A, Taggart J, Newall A, Denney-Wilson E, Zwar N, et al. Which providers can bridge the health literacy gap in lifestyle risk factor modification education: a systematic review and narrative synthesis. BMC Fam Pract. 2012;13:44. https://doi.org/10.1186/1471-2296-13-44

14. Silva-Júnior MF, Sousa MLR, Batista MJ. Health literacy on oral health practice and condition in a adult and elderly population. Health Promot Int. 2020:daaa135. https://doi.org/10.1093/heapro/daaa135

15. Kickbusch I, Pelikan JM, Apfel F, Tsouros AD, editors. Health literacy: the solid facts. Copenhagen (DK): WHO Regional Office for Europe; 2013 [citado 1 fev 2020]. Disponível em: https://apps.who.int/iris/bitstream/handle/10665/128703/e96854.pdf

16. World Health Organization. World report on ageing and health. Geneva (CH): WHO; 2015 [citado 7 out 2020]. Disponível em: http://apps.who.int/iris/bitstream/10665/186463/1/9789240694811_eng.pdf?ua=1

17. World Health Organization. Shanghai Declaration on promoting health in the 2030 Agenda for Sustainable Development. In: 9. Global Conference on Health Promotion; 2016 Nov 21-24; Shanghai, China. Geneva; WHO; 2016 [citado 7 out 2020]. Disponível em: https://www.who.int/healthpromotion/conferences/9gchp/shanghai-declaration.pdf

18. Firmino RT, Martins CC, Faria LS, Paiva SM, Granville-Garcia AF, Fraiz FC, et al. Association of oral health literacy with oral health behaviors, perception, knowledge, and dental treatment related outcomes: a systematic review and meta-analysis. J Public Health Dent. 2018;78(3):231-45. https://doi.org/10.1111/jphd.12266

19. Batista MJ, Marques ACP, Silva Junior MF, Alencar GP, Sousa MLR. Translation, cross-cultural adaptation and psychometric evaluation of Brazilian Portuguese version of the 14-item Health Literacy Scale. Cienc Saude Coletiva. 2020;25(7):2847-57. https://doi.org/10.1590/1413-81232020257.22282018 
20. Wang Y, McKee M, Torbica A, Stuckler D. Systematic literature review on the spread of health-related misinformation on social media. Soc Sci Med. 2019;240:112552. https://doi.org/10.1016/j.socscimed.2019.112552

21. Ho TG, Hosseinzadeh H, Rahman B, Sheikh M. Health literacy and health-promoting behaviours among Australian-Singaporean communities living in Sydney metropolitan area. Proc Singap Healthc. 2018;27(2):125-31. https://doi.org/10.1177/2010105817741906

22. Malta M, Cardoso LO, Bastos FI, Magnanini MMF, Silva CMFP. Iniciativa STROBE: subsídios para a comunicação de estudos observacionais. Rev Saude Publica. 2010;44(3):559-65. https://doi.org/10.1590/S0034-89102010000300021

23. Instituto Brasileiro de Geografia e Estatística. Censo demográfico 2010 - Cidades: panorama: Piracicaba. Rio de Janeiro: IBGE; 2010 [citado 15 mar 2019]. Disponível em: https://cidades.ibge.gov.br/brasil/sp/piracicaba/panorama

24. Secretaria Municipal de Saúde de Piracicaba. Plano Municipal de Saúde 2018-2021. Piracicaba, SP: SMS; 2017 [citado 23 mar 2018]. Disponível em: http://saude.piracicaba.sp.gov.br/institucional/ppa-plano-plurianual/

25. Perez Puello SC. Avaliação da literacia em saúde [dissertação]. Piracicaba, SP: Universidade Estadual de Campinas, Faculdade de Odontologia de Piracicaba; 2018.

26. Morgan BS. Avaliação do monitoramento telefônico na promoção do autocuidado em diabetes na atenção primária em saúde [dissertação]. Belo Horizonte, MG: Universidade Federal de Minas Gerais, Escola de Enfermagem; 2013.

27. Ainamo J, Bay I. Problems and proposals for recording gingivitis and plaque. Int Dent J. 1975;25 (4):229-35.

28. Organização Mundial da Saúde; Faculdade de Odontologia da Universidade de São Paulo. Levantamentos em Saúde Bucal: métodos Básicos. 5. ed. São Paulo: FOUSP; 2017 [citado 7 jan 2019]. Disponível em: http://www.fo.usp.br/wp-content/uploads/2011/06/ Levantamentos-Saude-Bucal_5ed_Nov2017.pdf

29. Frias AC. Estudo de confiabilidade do Levantamento Epidemiológico de Saúde Bucal - Estado de São Paulo 1998 [dissertação]. São Paulo: Universidade de São Paulo, Faculdade de Saúde Pública; 2000.

30. Silva-Junior MF, Sousa MR, Batista MJ. Prospective cohort of adult oral health in Piracicaba, SP, Brazil. BMC Res Notes. 2019;12:221. https://doi.org/10.1186/s13104-019-4243-y

31. Suka $M$, Odajima $T$, Kasai $M$, Igarashi $A$, Ishikawa $H$, Kusama $M$, et al. The 14-item health literacy scale for Japanese adults (HLS-14). Environ Health Prev Med. 2013;18:407-15. https://doi.org/10.1007/s12199-013-0340-z

32. Quartuccio M, Simonsick EM, Langan S, Harris T, Sudore RL, Thorpe R, et al. The relationship of health literacy to diabetes status differs by sex in older adults. J Diabetes Complications. 2018;32(4):368-72. https://doi.org/10.1016/j.jdiacomp.2017.10.012

33. Ministério da Saúde (BR), Seretaria de Atenção à Saúde, Secretaria de Vigilância em Saúde. SB Brasil 2010: Pesquisa Nacional de Saúde Bucal: resultados principais. Brasília, DF; 2012 [citado 29 abr 2019]. Disponível em: https://bvsms.saude.gov.br/bvs/publicacoes/pesquisa_nacional_saude_bucal.pdf

34. Queres JFM, Lima BA, Camara MPM, Santos MCC, Taboada GF. Avaliação do conhecimento e atitudes e sua relação com parâmetros de controle do diabetes mellitus. J Health Connec. 2018;2(1):21-9.

35. Ministério da Saúde (BR), Secretaria de Atenção à Saúde, Departamento de Atenção Básica. Estratégias para o cuidado da pessoa com doença crônica: diabetes mellitus. Brasília, DF; 2013 [citado 29 abr 2019]. (Cadernos de Atenção Básica; n 36). Disponível em: https://bvsms. saude.gov.br/bvs/publicacoes/estrategias_cuidado_pessoa_diabetes_mellitus_cab36.pdf

36. Ministério da Saúde (BR). Secretaria de Atenção à Saúde, Departamento de Atenção Básica. Estratégias para o cuidado da pessoa com doença crônica: hipertensão arterial sistêmica. Brasília, DF; 2013 [citado 29 abr 2019]. (Cadernos de Atenção Básica; nº 37). Disponível em: https://bvsms.saude.gov.br/bvs/publicacoes/estrategias_cuidado_pessoa_doenca_cronica.pdf

37. Armellini D, Fraunhofer JA. The shortened dental arch: a review of the literature. J Prosthet Dent. 2002;92(6):531-5. https://doi.org/10.1016/j.prosdent.2004.08.013

38. Nutbeam D. Health literacy as a public health goal: a challenge for contemporary health education and communication strategies into the 21 st century. Health Promot Int. 2000;15(3):259-67. https://doi.org/10.1093/heapro/15.3.259 
39. Sørensen K, Van den Broucke S, Fullam J, Doyle G, Pelikan J, Slonska Z, et al. Health literacy and public health: a systematic review and integration of definitions and models. BMC Public Health. 2012;12:80. https://doi.org/10.1186/1471-2458-12-80

40. Martins AMEBL, Almeida ER, Oliveira CC, Oliveira RCN, Pelino JEP, Santos ASF, et al. Alfabetização em saúde bucal: uma revisão da literatura. Rev Assoc Paul Cir Dent. 2015;69(4):328-34.

41. Andersen RM, Davidson PL. Ethnicity, aging, and oral health outcomes: a conceptual framework. Adv Dent Res. 1997;11(2):203-9. https://doi.org/10.1177/08959374970110020201

42. Yadav UN, Hosseinzadeh H, Lloyd J, Harris MF. How health literacy and patient activation play their own unique role in self-management of chronic obstructive pulmonary disease (COPD)? Chron Respir Dis. 2019;16:1479973118816418. https://doi.org/10.1177/1479973118816418

43. Henderson E, Dalawari P, Fitzgerald J, Hinyard L. Association of oral health literacy and dental visitation in an inner-city emergency department population. Int J Environ Res Public Health. 2018;15(8):1748. https://doi.org/10.3390/ijerph15081748

44. Batista MJ, Lawrence HP, Sousa MRL. Oral health literacy and oral health outcomes in an adult population in Brazil. BMC Public Health. 2018;18(1):60. https://doi.org/10.1186/s12889-017-4443-0

45. Firmino RT, Ferreira FM. Paiva SM, Granville-Garcia AF, Fraiz FC, Martins CC. Oral health literacy and associated oral conditions: a systematic review. J Am Dent Assoc. 2017;148(8):604-13. https://doi.org/10.1016/j.adaj.2017.04.012

46. Gomes Filho VV, Gondinho BVC, Silva-Junior MF, Cavalcante DFB, Bulgareli JV, Sousa $M R L$, et al. Tooth loss in adults: factors associated with the position and number of lost teeth. Rev Saude Publica. 2019;53:105. https://doi.org/10.11606/S1518-8787.2019053001318

47. Helal O, Göstemeyer G, Krois J, El Sayed KF, Graetz C, Schwendicke F. Predictors for tooth loss in periodontitis patients: systematic review and meta-analysis. J Clin Periodontol. 2019;46(7):699-712. https://doi.org/10.1111/jcpe.13118

48. Watt RG. From victim blaming to upstream action: tackling the social determinants of oral health inequalities. Community Dent Oral Epidemiol. 2007;35(1):1-11. https://doi.org/10.1111/j.1600-0528.2007.00348.x

49. Solar $\mathrm{O}$, Irwin A. A conceptual framework for action on the social determinants of health. Geneva (CH): WHO; 2010. (Social Determinants of Health Discussion Paper; $n^{\circ} 2$ : Policy and Practice).

50. Cabellos-García AC, Castro-Sánchez E, Martínez-Sabater A, Díaz-Herrera MA, Ocaña-Ortiz A, Juárez-Vela R, et al. Relationship between determinants of health, equity, and dimensions of health literacy in patients with cardiovascular disease. Int J Environ Res Public Health. 2020;17(6):2082. https://doi.org/10.3390/ijerph17062082

51. Maia FBM, Sousa ET, Sampaio FCS, Freitas CHSM, Forte FDS. Tooth loss in middle-aged adults with diabetes and hypertension: social determinants, health perceptions, oral impact on daily performance (OIDP) and treatment need. Med Oral Patol Oral Cir Bucal. 2018;23(2):e203-10. https://doi.org/10.4317/medoral.22176

52. Kocher T, König J, Borgnakke WS, Pink C, Meisel P. Periodontal complications of hyperglycemia/diabetes mellitus: epidemiologic complexity and clinical challenge. Periodontology. 2000;78(1):59-97. https://doi.org/10.1111/prd.12235

53. Marmot M, Allen J, Bell R, Bloomer E, Goldblatt P, et al. WHO European review of social determinants of health and the health divide. Lancet. 2012;380(9846):1011-29. https://doi.org/10.1016/S0140-6736(12)61228-8

54. Michaud DS, Fu Z, Shi J, Chung M. Periodontal disease, tooth loss, and cancer risk. Epidemiol Rev. 2017;39(1):49-58. https://doi.org/10.1093/epirev/mxx006

55. Dörfer C, Benz C, Aida J, Campard G. The relationship of oral health with general health and NCDs: a brief review. Int Dent J. 2017;67 Suppl 2:14-8. https://doi.org/10.1111/idj.12360

56. Lee HJ, Choi EK, Park JB, Han KD, Oh S. Tooth loss predicts myocardial infarction, heart failure, stroke, and death. J Dent Res. 2019;98(2):64-70. https://doi.org/10.1177/0022034518814829

57. Genco RJ, Sanz M. Clinical and public health implications of periodontal and systemic diseases: an overview. Periodontology 2000;2020;83(1):7-13. https://doi.org/10.1111/prd.12344

58. Friedman PK, Lamster IB. Tooth loss as a predictor of shortened longevity: exploring the hypothesis. Periodontology 2000;2016;72(1):142-52. https://doi.org/10.1111/prd.12128 
59. Salci MA, Silva DMGV, Meirelles BHS, Rêgo AS, Radovanovic CAT, Carreira L, et al. Diabetes mellitus e saúde bucal: a complexa relação desta assistência na atenção primária à saúde. Saude Pesq. 2020;13(2):265-72. https://doi.org/10.1111/prd.12128

60. Kassebaum NJ, Smith AG, Bernabé E, Fleming TD, Reynolds AE, Vos T, et al. Global, regional, and national prevalence, incidence, and disability-adjusted life years for oral conditions for 195 countries, 1990-2015: a systematic analysis for the Global Burden of Diseases, injuries, and risk factors. J Dent Res. 2017;96(4):380-7. https://doi.org/10.1177/0022034517693566

61. GBD 2019 Universal Health Coverage Collaborators. Measuring universal health coverage based on an index of effective coverage of health services in 204 countries and territories, 1990-2019: a systematic analysis for the Global Burden of Disease Study 2019. Lancet. 2020;396(10258):1250-84. https://doi.org/10.1016/S0140-6736(20)30750-9

62. Barreto ACO, Rebouças CBA, Aguiar MIF, Barbosa RB, Rocha SR, Cordeiro LM, et al. Perception of the Primary Care multiprofessional team on health education. Rev Bras Enferm. 2019;72 Suppl 1:266-73. https://doi.org/10.1590/0034-7167-2017-0702

Contribuição dos Autores: Concepção e planejamento do estudo: CFT, MJB. Coleta, análise e interpretação dos dados: CFT, CML, MJB. Elaboração ou revisão do manuscrito: CFT, MFSJ, CML, MLRS, MJB. Aprovação da versão final: CFT, MJB, MFSJ, MLRS. Responsabilidade pública pelo conteúdo do artigo: CFT, MFSJ, CML, MLRS, MJB.

Conflito de Interesses: Os autores declaram não haver conflito de interesses. 